\title{
Educación Interprofesional: un desafío para dar visibilidad a la contribución de Enfermería en los equipos de salud
}

\author{
Interprofessional Education: a challenge \\ to give visibility to the contribution \\ of Nursing in health teams
}

\section{Educação interprofissional: um desafio para dar visibilidade à contribuição da Enfermagem nas equipes de saúde}

La Educación Interprofesional (EIP) en salud posee varias definiciones que se complementan y aportan diferentes enfoques. Según la OMS esta se define como: un enfoque de enseñanza y aprendizaje que reúne a estudiantes de dos o más profesiones para aprender juntos, con el fin de mejorar la colaboración y los resultados en salud ${ }^{1}$. El Centre for the Advancement of Interprofessional Education, (CAIPE) menciona que esta ocurre cuando dos o más profesiones aprenden entre sí, con y sobre las otras, para mejorar la colaboración y la calidad de los cuidados².

La Canadian Interprofessional Health Collaborative señala que la EIP está presente cuando los profesionales de la salud aprenden colaborativamente dentro y entre las disciplinas, a fin de obtener conocimientos, habilidades y valores necesarios para trabajar con otros profesionales de la salud 3 .

Finalmente, la Interprofessional Education Collaborative Expert Panel (IPEC) la plantea como un proceso de aprendizaje que prepara a los profesionales a través de la educación interdisciplinaria y de las diversas experiencias de la realidad del trabajo en salud, en colaboración con las comunidades para atender las necesidades multifacéticas de niños, jóvenes y familias 4 .

Estos planteamientos se aglutinan bajo la premisa que une a todas estas organizaciones internacionales aprender juntos para trabajar juntos a través de las prácticas colaborativas en salud, con los que se inicia un movimiento global que promueve dicha estrategia. En la actualidad se cuenta con mayores fortalezas conceptuales y propuestas metodológicas para el desarrollo de competencias para trabajar en equipo con mayor eficiencia y eficacia, esto permite mejorar la calidad del cuidado y los servicios de salud. Se han conformado múltiples redes para colaborar en el fortalecimiento de la 
estrategia, por ejemplo, intercambiar experiencias, materiales y actividades de formación, consolidándose como camino para fortalecer la lógica de la colaboración en la dinámica del trabajo en salud, según lo planteado por Reeves 5 .

La EIP surge como respuesta a los problemas actuales en los sistemas de salud y en la búsqueda de estrategias de enseñanza que regulen los cambios estructurales de los servicios y los procesos que implica el cuidado a la salud. Involucra una concepción integral y abarcativa del concepto salud que requiere la superación del modelo de formación de los silos profesionales ${ }^{6}$ y que conlleva a una reforma de la enseñanza en salud para la mejora del cuidado a la salud de la población; integra en ese concepto la necesaria conjunción entre la enseñanza, la investigación, así como el aporte a la sociedad a través del cuidado de la salud y la interacción con los equipos profesionales de los servicios sanitarios.

La incorporación del usuario como centro del sistema de atención a la salud es otro concepto a integrar en la transformación del funcionamiento del equipo y de los resultados obtenidos.

Los problemas concretos que se propone resolver la estrategia de EIP y del trabajo colaborativo en salud son: duplicación de los actos profesionales con la consecuente insatisfacción de los usuarios y mal uso de los recursos humanos y financieros; falta de integración y articulación de los roles profesionales para lograr mayor capacidad de resolución en los problemas de salud de la población; la expansión de la jurisdicción de algunos campos profesionales superpuestos con los de otros profesionales; los errores en la atención por dificultades en la comunicación y conflictos internos en los equipos; aumento de costos de los servicios de salud; falta de cobertura de grupos de usuarios con perfiles epidemiológicos diferentes que surgen con los cambios demográficos actuales; y las expectativas e información que poseen los usuarios.

Aun cuando los campos profesionales se han visto remodelados y han redefinido los límites internos de la profesión, persisten al formar parte del todo en los equipos de salud, con conflictos, resistencias y oportunidades para todos y cada uno de ellos. Por lo tanto, cada vez resulta más necesario el trabajo colaborativo en salud para lo cual las disciplinas deben tener claridad, consistencia y especificidad para aportar a ese todo (es decir, los equipos). A la par deben fortalecer la capacidad de negociar las funciones y compartir las potencialidades que surgen de la mencionada remodelación de los campos profesionales; ampliar los roles profesionales implica modificar la cultura de trabajo de sus componentes y modificar las culturas de trabajo de las organizaciones. El manejo del poder al interior de los equipos, las imágenes sociales, históricas y la cultura de las profesiones, están transformándose de forma vertiginosa, al tiempo que es imperioso que se ajusten a las transformaciones de las otras profesiones y a la vez se articulen entre sí.

Surge así la necesidad de re pensar y analizar críticamente, reformular el rol que juega el personal de enfermería en esos equipos, de conocer cuáles son los aportes que realizan como profesión, qué esperan de su colaboración otros profesionales y la población usuaria de los servicios, en contraposición con los cambios que la profesión está procesando y reformulando. La forma de avanzar y lograr sus objetivos, en paralelo con cambios que mejoren la calidad del cuidado a la salud de las poblaciones, serán producto de reflexión y discusiones colectivas.

La Enfermería ha definido desde muchos años atrás, el cuidado como objeto disciplinar. Dado que es un objeto compartido y abarcativo, es también un espacio de disputa de varios profesionales, cruzado por diferenciaciones de género, clases sociales, prestigio social, historia. Cuidar y curar forman parte del espacio profesional de todas las profesiones de la salud, delimitar las especificidades para cada uno sin descuidar el sumar al todo, ahí está el gran desafío. 
Para Enfermería el trabajo en equipo es parte necesaria del trabajo cotidiano, éste se fundamenta en principios tales como la continuidad del cuidado a lo largo del ciclo vital, la articulación con todos los integrantes del equipo, la función de coordinación, el acceso a información relevante de los usuarios por su cercanía con las personas (objeto de cuidado), es la única profesión miembro del equipo que posee un integrante al cuidado de los usuarios en forma continua (lo que requiere un equipo de enfermería en sí). Por lo tanto, las condiciones de integración a equipos colaborativos están basadas en una cultura profesional de trabajo en equipo arraigada y que es parte de la vida profesional.

El desafío mayor está dado por la innovación que requiere la enseñanza con la modalidad participativa de la EIP en los equipos docentes de diferentes disciplinas que elaboren programas, planes, actividades, experiencias de carácter interprofesional, con las características y recursos disponibles a su alcance para integrar a los estudiantes desde su formación básica. Esta estrategia implica necesarias negociaciones de contenidos, metodologías, elección de temáticas de todos los docentes participantes en un vínculo donde los docentes desarrollen una comunicación asertiva entre sí y con otros (comunicación horizontal), donde haya resolución de conflictos al interior de los equipos, difusión de la estrategia, así como la inclusión en el plan de estudios de las actividades planificadas que serán un ensayo para consolidar las experiencias a desarrollar con los estudiantes a posteriori.

La EIP implica una oportunidad para que enfermería avance en dar visibilidad a sus potencialidades disciplinares en el intercambio con otras profesiones, tanto en los docentes como en la formación de los futuros profesionales, al difundir cuál es el potencial en habilidades blandas y en cuidados que puede aportar a los equipos, desde el ejercicio autónomo que eleve su autoestima profesional, valorizándose a sí mismo y a su colectivo al tiempo que se integra al aprendizaje que genera el trabajo colaborativo, el intercambio de experiencias de aprendizaje conjuntas.

Es imprescindible dar inicio progresivo a actividades que promuevan la EIP, que implica capacitación de los docentes para desarrollar programas, planes de estudio, organizar actividades conjuntas? acuerdos entre diferentes instituciones formativas y el conocimiento de la realidad asistencial, así como las necesidades de la población.

La comprensión por parte de los docentes del concepto, el valor y la importancia de la EIP, favorecerá la implementación de las actividades y acuerdos, para resolver las barreras y conflictos que provienen de las transformaciones del poder, el abandono de la zona de confort profesional (siempre lo hicimos asi) normal y esperable en cualquier cambio.

Implica a la vez, por una parte, crecimiento y transformación profesional para mejorar la calidad de vida en el trabajo de Enfermería, donde exista mayor satisfacción y reconocimiento de la profesión, sus potencialidades y capacidades. Y por otra, como plantea la premisa de la EIP, aprender juntos para trabajar juntos con el cometido de brindar la mejor calidad de atención posible a la población, de forma que el personal de enfermería como mayor fuerza de trabajo en salud, colaboren y sean impulsores de los cambios necesarios para lograr la cobertura universal de la salud, los objetivos del milenio y la necesidad de avanzar continuamente hacia la excelencia.

En ese sentido, como señala Azita Emami (Directora de la Escuela de Enfermería de la Universidad de Washington, UWSON, con amplia experiencia en el desarrollo de programas de formación con enfoque de EIP): La práctica clínica, la educación y la investigación interprofesional nos permiten preparar la próxima generación de equipos de profesionales de la salud en un camino necesario a recorrer para lograr esa excelencia. 


\section{REFERENCIAS}

1. World Health Organization/Health Professions Networks Nursing/Midwifery Human Resources for Health. Framework for action on interprofessional education \& collaborative practice. Geneva, Switzerland: WHO; 2010. http://bit.ly/2VOpeTX

2. Red Regional de Educación Interprofesional de las Américas. Qué es la educación interprofesional. REIP. Washington, D.C.: VCPH, PAHO; 2018. http://bit.ly/2px3L6I

3. Canadian Interprofessional Health Collaborative. A National Interprofessional Competency Framework. Vancouver, Canada: College of Health Disciplines, University of British Columbia; 2010. http://bit.ly/32mM3RS

4. Interprofessional Education Collaborative. Core competencies for Interprofessional Collaborative Practice, Report of an Expert Panel. Washington, D.C.: IPEC; 2011. http://bit.ly/2IYDTaE

5. Barr H, Low H. Introducción a la Educación Interprofesional. Reino Unido: CAIPE; 2013. http://bit.ly/2MmAepc

6. Costa-Viana da $M$, Peduzzi $M$, Freire Filho-Rodrigues J, Silva-Brandão Gonçalves C. Educación Interprofesional en Salud. Natal/RN, Brasil: SEDIS-UFRN; 2018. http://bit.ly/2Mo4kYf

7. Beunza-Nuin JJ, Icarán-Francisco E. Manual de Educación Interprofesional Sanitaria. España: Elsevier; 2018.

M. Pérez-Etchetto

ORCID: 0000-0001-9963-3630 Decana de Facultad de Enfermería Universidad de la República Uruguay decanato@fenf.edu.uy 